that important subsets of patients experience inadequate disease control with current therapies. This study reveals high-dose corticosteroid use is prevalent in SLE management broadly, underscoring the unmet need in this population.

Disclosure of Interest: K. Schroeder Shareholder of: Eli Lilly and Company, Employee of: Eli Lilly and Company, J. Paik Shareholder of: Eli Lilly and Company, Employee of: Eli Lilly and Company, R. Hoffman Shareholder of: Eli Lilly and Company, Employee of: Eli Lilly and Company, S. Gelwicks: None declared DOI: 10.1136/annrheumdis-2018-eular.1921

\section{THU0375 QUALITY OF LIFE IN INDIAN PATIENTS WITH SYSTEMIC LUPUS ERYTHEMATOSUS IN DURABLE REMISSION: PSYCHOSOCIAL AND DEMOGRAPHIC FACTORS}

${ }^{1}$ R.P. Goswami, R. Chatterjee ${ }^{2}$, A. Mitra ${ }^{2}$, B. Bhattacharya ${ }^{2}$, A. Ghosh ${ }^{1}$, P. Ghosh ${ }^{1}$. ${ }^{1}$ Department of Rheumatology, IPGMER, Kolkata, India, Institute of Post Graduate Medical Education and Research; ${ }^{2}$ Department of Clinical Psychology, Institute of Psychiatry, Kolkata, India

Background: Remission in systemic lupus erythematosus (SLE) is uncommon. Detrimental effect of disease activity on quality of life (QoL) is reported but literature on QoL in lupus patients in durable remission is scant.

Objectives: To study QoL in Indian SLE patients in durable remission

Methods: We retrospectively included female SLE patients fulfilling $\geq 4$ SLICC Classification Criteria, followed regularly at our clinic, who were in durable remission as determined by European consensus criteria (complete/clinical remission timmunosuppressive drugs). QoL was assessed with Medical Outcomes Study Short-Form-12 (SF-12). We also collected data on demographics (age, duration of disease, years of education), duration and quality (complete versus clinical) of remission and patient reported fatigue through fatigue severity scale (FSS). A structured interview with a clinical psychologist using ICD-10 Diagnostic Criteria for Research (DCR) was performed to diagnose depression. Age matched female control subjects were also included and underwent similar exercises. Association of physical and mental component summary scores (PCS and MCS) of SF-12 with depression, quality and duration of remission, duration of disease, years of education and FSS were tested with generalised linear models using Gamma regression with log-link function.

Results: We included 106 female SLE patients (age: $28.9 \pm 7.6$ years; duration of disease: $45.1 \pm 34.8$ months; years of education: $9.6 \pm 5.2$; depression present in 41 $(38.7 \%)$ ) and 98 female controls (age: $30.4 \pm 7$ years; years of education: 10.8 \pm 6.2 ; depression present in $32(32.7 \%))$. At last visit, clinical remission was present in $68(64.2 \%)$ and complete remission in $38(35.2 \%)$. Duration of remission achieved were:<1 year in $17(16 \%), 1-2$ years in $40(37.7 \%), 2-3$ years in 18 $(17 \%)$ and $>3$ years in $31(29.2 \%)$. Steroid-free remission was present in 64 $(60.37 \%)$ and the rest $42(39.62 \%)$ were on $\leq 5 \mathrm{mg} / \mathrm{d}$ prednisolone. All were on hydroxychloroquine. A stable dose of 2 nd immunosuppressive drug was present in $54(50.94 \%)$ with 3 on stable dose of mycophenolate and 51 on azathioprine. SLE patients had comparable SF-12 PCS (48.4 \pm 8.1 vs $47.1 \pm 7, \mathrm{p}=0.098)$ and MCS $(57.7 \pm 3.4$ vs $56.6 \pm 5.6, p=0.199)$ as compared to controls. Among lupus patients, both PCS $(r=-0.616, p<0.001)$ and MCS $(r=-0.298, p<0.001)$ were correlated with FSS and years of education (with PCS, $r=-0.215, p=0.027$; with MCS, $r=-0.269, p=0.005$ ). Independent predictors of PCS were: clinical remission (Odd's ratio (OR) $0.95,95 \%$ confidence interval $(\mathrm{Cl}) 0.92-0.99, p=0.033$ ), FSS (OR $0.90,95 \% \mathrm{Cl} 0.89-0.92, \mathrm{p}<0.001)$ and disease duration $<5$ years (OR $0.92,95 \% \mathrm{Cl} 0.86-0.97, \mathrm{p}=0.006)$. Independent predictors of MCS were: FSS (OR $0.991,95 \% \mathrm{Cl} 0.983-0.999, \mathrm{p}=0.025$ ), years of education (OR 0.998, $95 \% \mathrm{Cl}: 0.995-0.999, \mathrm{p}=0.028)$ and disease duration $<5$ years (OR 1.053, $95 \% \mathrm{Cl} 1.018-1.089, \mathrm{p}=0.003)$. Estimated marginal means of PCS and MCS against quality of remission and duration of disease are plotted in figure 1.

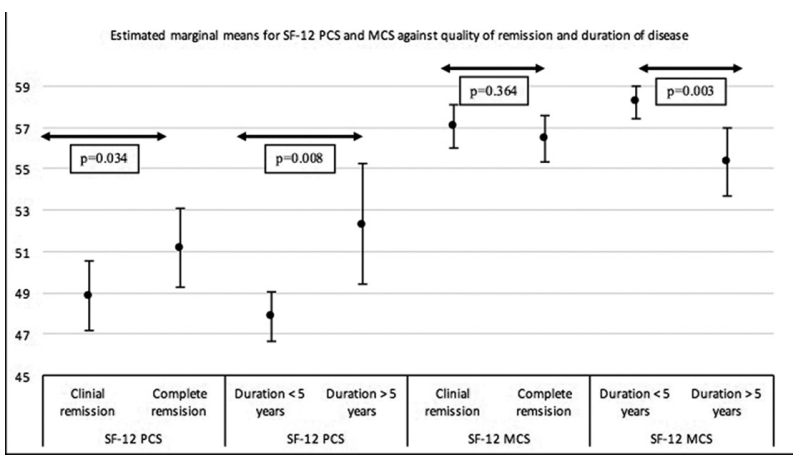

Abstract THU0375 - Figure 1. Estimated marginal means for SF-12 PCS and MCS against quality of remission and duration of disease
Conclusions: Indian lupus patients in durable remission had similar physical and mental QoL compared to healthy controls. Physical QoL was better in patients with complete remission, longer disease duration and low fatigue. Mental QoL was better in patients with low fatigue, less education and longer disease duration.

Disclosure of Interest: None declared

DOI: 10.1136/annrheumdis-2018-eular.2536

\section{THU0376 \\ CHARACTERISTICS OF PRIMARY SJÖGREN'S SYNDROME PATIENTS WITH MORPHOLOGICAL CHANGES OF THE PAROTID GLANDS IN MR IMAGING}

${ }^{1}$ S. Zhao, H. Zhang ${ }^{1}$, C. Chu ${ }^{2}$, X. Feng ${ }^{1}$, L. Sun ${ }^{1} .{ }^{1}$ Department of Rheumatology and Immunology; ${ }^{2}$ Department of Radiology, The Affiliated Drum Tower Hospital of Nanjing University Medical School, Nanjing, China

Background: Primary Sjögren's syndrome (pSS) is a chronic autoimmune disease characterised by injury of exocrine glands, and a considerable proportion of pSS patients develop extraglandular involvement. The parotid glands are the most frequently involved glands in pSS. Conventional parotid examinations, such as X-Ray sialography and 99mTechnetium ([99mTc]) pertechnetate scintigraphy, played an important role in the diagnosis of pSS. However, X-Ray sialography only shows the abnormality of parotid ductal system and there is exposure to radionuclides with $99 \mathrm{mTechnetium.} \mathrm{Both} \mathrm{examinations} \mathrm{are} \mathrm{invasive,} \mathrm{while} \mathrm{MR}$ imaging is noninvasive, radiation-free, and sensitive to the morphological and signal changes of the parotid glands. MR sialography could be used to evaluate the parotid ductal system without the need for a contrast agent. But the clinical application value of parotid grand MR imaging in pSS patients has not been verified. Objectives: The purpose of this study was to investigate the morphological changes of the parotid glands in MR imaging in patients with pSS and the correlations between morphological changes and the clinical manifestations.

Methods: Ninty-nine pSS patients who underwent parotid 3.0 Tesla MR imaging (T1, T2 and T2 STIR) were enrolled in this study. The morphological changes of the parotid glands (grades $0-3$ ) and ducts (grades $0-4$ ) were rated according to our previous studies. ${ }^{1}$ Patients were divided into normal parotid MR group (both glands grade and ducts grade were 0 ) and abnormal parotid MR group. The correlations between morphological changes of the parotid glands and clinical or serological characteristics were analysed by chi-square test.

Results: There were 93 females $(93.9 \%)$ and 6 males $(6.1 \%)$ in this study. The mean age and median disease duration were 47.4 years and 24 months. There were $50(50.5 \%)$ pSS patients in parotid grand grade $0,27(27.3 \%)$ in grade 1,15 $(15.2 \%)$ in grade 2 and $6(6.1 \%)$ in grade 3 (Fig $1 \mathrm{~A})$, and there were $53(53.5 \%)$ pSS patients in parotid duct grade $0,15(15.2 \%)$ in grade $1,17(17.2 \%)$ in grade 2 , $4(4.0 \%)$ in grade 3 , and $10(10.1 \%)$ in grade 4 (Fig 1B). We found that patients in abnormal parotid MR group presented lower positive rates of myasthenia and higher positive rates of xerostomia, Schimer's test, serum anti-SSA antibodies, anti-Ro-52 antibodies, antinuclear antibodies (ANA), rheumatoid factor (RF), plasma globulin, immunoglobulin $G(\lg G)$, and Hashimoto thyroiditis $(p<0.05)$. But no significant difference was observed between two groups in the incidence of salivary gland enlargement, articular involvement, dermatological involvement, interstitial lung disease, tubulointerstitial nephritis and primary biliary cirrhosis.
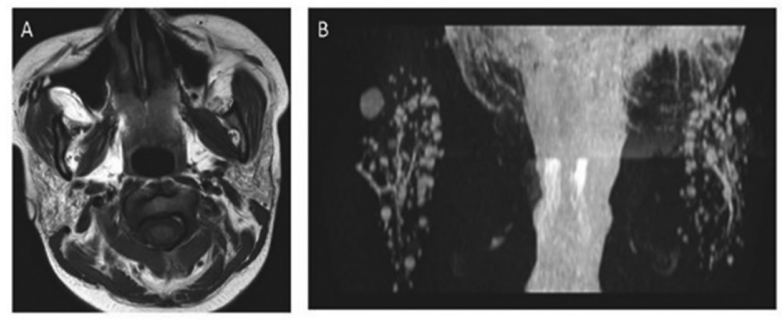

Abstract THU0376 - Figure 1. A: Axial T1-weighted image shows homogenous signal intensity of bilateral parotid glands with obvious hyperintense nodules (grade 3). B: MR sialography shows duct dilation of bilateral parotid glands (grade 4).

Conclusions: The results indicated that parotid MR imaging is a noninvasive radiation-free examination with a potential role in diagnosing pSS. pSS patients with morphological changes of the parotid glands in MR imaging were more likely to have xerostomia, hyperglobulinemia and thyroid involvement. 


\section{REFERENCE:}

[1] Chen Chu, Nan Zhou, Huayong Zhang, et al. Added Value of Parotid R2* Values for Evaluation of Sjögren Syndrome: A Preliminary Study. J Comput Assist Tomogr 2017;41(4):547-552.

Disclosure of Interest: None declared

DOI: 10.1136/annrheumdis-2018-eular.6214

\section{THU0377 INFLUENCE OF UNDIAGNOSED VERTEBRAL FRACTURES ON ORGAN DAMAGE IN WOMEN WITH SYSTEMIC LUPUS ERYTHEMATOSUS}

\begin{abstract}
${ }^{1,2}$ S. Shkireeva, O.M. Lesnyak ${ }^{1}$, E.G. Zotkin' ${ }^{1}{ }^{1}$ North-Western State Medical University named after I.I. Mechnikov, Saint-Petersburg; ${ }^{2}$ Research Institute of Rheumatology named after V.A. Nasonova, Moscow, Russian Federation
\end{abstract}

Objectives: To investigate the influence of asymptomatic vertebral fractures on organ damage and to identify risk factors associated with critical organ damage in women with systemic lupus erythematosus (SLE).

Methods: 197 women with SLE were included in this study. Bone mineral density (BMD) measurements of the hip and spine were performed using the dual energy X-ray absorptiometry (DXA). Vertebral fracture assessment (VFA) was done for detection vertebral fractures using a method described by Genant. Accumulated damage was scored using the SLICC/ACR damage index (SDI). Critical organ damage was defined as $\mathrm{SDI}>3$.

Results: Vertebral fractures were developed in 55 (27.9\%) women with SLE. Half of all women with SLE $(n=31,15.7 \%)$ had asymptomatic vertebral fractures which were diagnosed for the first time in this study. 131 (66.5\%) women with SLE had critical organ damage (SDI>3). Average SDI before and after morphometry was $4,4 \pm 2,2$ and $5,3 \pm 2,6$ respectively. Multivariate analysis showed age $(p=0,01)$, cumulative dose of glucocorticoids ( $p=0,00005)$, previous therapy with cyclophosphamide $(p=0,04)$ were significantly associated with critical damage in women with SLE.

Conclusions: Detection of vertebral fractures helps in counting accumulated organ damage correctly. VFA in the combination with DXA in women with SLE is an effective method for diagnostic asymptomatic vertebral fractures.

Disclosure of Interest: None declared

DOI: 10.1136/annrheumdis-2018-eular.5462

\section{THU0378 WIRE-LOOP LESION IS ASSOCIATED WITH SEROLOGICAL IMMUNE ABNORMALITY, BUT NOT RENAL PROGNOSIS IN LUPUS NEPHRITIS}

T. Zoshima, S. Hara, M. Kawano. Division of Rheumatology, Kanazawa University Hospital, Kanazawa, Japan

Background: International Society of Nephrology/Renal Pathology Society (ISN/ RPS) 2003 Classification of Lupus Nephritis (LN) defines wire-loop lesion (WL) as an active lesion (AL). In it, LN patients with WL are classified as class III or IV, which are associated with a poor prognosis and recommended to be treated by intense immunosuppressive therapy including corticosteroid, cyclophosphamide, mycophenolate mofetil and other immunosuppressants. 'However, among AL, few reports have focused on the clinicopathological impact of WL on serological immune abnormality and renal prognosis.

Objectives: To identify clinicopathological characteristics associated with WL, and to clarify whether WL predicts renal prognosis of $L N$.

Methods: We enrolled 117 Japanese LN patients subjected to renal biopsy in 11 hospitals from 2000 to 2017. We measured clinical findings at the time of renal biopsy, including creatinine ( $\mathrm{Cr}$ ), estimated glomerular filtration rate (eGFR), total protein (TP), IgG, IgA, IgM, C3, C4, CH50, anti-nuclear antibodies (Abs), anti-double strand DNA (dsDNA) Abs, anti-Sm Abs, anti-RNP Abs in the sera, urinalysis, and presence of comorbidities (antiphospholipid antibody syndrome, hypertension, hyperlipidemia, diabetes mellitus, and hyperuricemia). Renal biopsy findings were classified by ISN/RPS classification including AL and chronic lesions (CL). Immune deposit was evaluated by immunofluorescence. We also measured $\mathrm{Cr}$ and eGFR at the last patient visit, and recorded medications prescribed for LN. Chronic kidney disease (CKD) was defined as eGFR $<60 \mathrm{ml} / \mathrm{min} / 1.73 \mathrm{~m} 2$. In class III or IV patients, we retrospectively compared these clinical and histological findings between those with WL (WL + group) and without WL (WL- group).

Results: Of 117 patients, 94 (81.2\%) were classified as class III or IV (78 females; mean age 41.3 years; observational period $5.8 \pm 5.2$ years). WL was found in 27 of them $(28.7 \%)$. Although there was no significant difference in renal function (eGFR; $81.1 \pm 31.4$ vs $80.6 \pm 34.4 \mathrm{ml} / \mathrm{min} / 1.73 \mathrm{~m} 2, \mathrm{p}=0.91$ ), WL + group had higher titer of serum anti-dsDNA Abs (median values; 205 vs $67 \mathrm{lU} / \mathrm{ml}, \mathrm{p}=0.011$ ) and lower level of TP $(5.7 \pm 1.2$ vs $6.3 \pm 1.0 \mathrm{~g} / \mathrm{dl}, \mathrm{p}=0.025)$ than $\mathrm{WL}$ - group. There were no significant differences in any other clinical findings. In histological findings, most WL (96.3\%) were accompanied by other ALs such as endocapillary proliferation and/or crescent formation. WL + group had a higher frequency of class IV $\mathrm{AL}$ and IgM deposit, while $\mathrm{CL}$ did not differ between the two groups. Linear regression analysis revealed associations between anti-dsDNA Abs and IgM deposit and $W L(\beta=0.48, p<0.01 ; \beta=0.26, p=0.016$, respectively). There was no difference in the latest renal function (eGFR; $76.9 \pm 27.5 \mathrm{vs} 74.0 \pm 29.5 \mathrm{ml} / \mathrm{min} / 1.73 \mathrm{~m} 2$, $\mathrm{p}=0.72$ ) between the two groups. Cox regression analysis revealed significant associations between AL such as necrosis, CL, initial eGFR, HT, HL and medication for $L N$ with CKD at the last visit, but not with WL $(p=0.13)$

Conclusions: WL was associated with serum anti-dsDNA Abs, but not with renal prognosis, suggesting that WL reflects immune abnormality, but is not an independent factor predictive of the renal prognosis of $L N$. Although $W L$ is defined as an $\mathrm{AL}$ in the present classification, this may need to be revised to better reflect its clinical impact.

\section{REFERENCE:}

[1] Ann Rheum Dis 2012;71:1771-82.

Disclosure of Interest: None declared

DOI: 10.1136/annrheumdis-2018-eular.4408

\section{THU0379 CORONARY ARTERY DISEASE IN SLE: A CASE- CONTROLLED ANGIOGRAPHIC STUDY}

${ }^{1}$ T. Chazal, M. Kerneis ${ }^{2}$, P. Guedeney ${ }^{2}$, J. Haroche ${ }^{1,3}$, A. Mathian ${ }^{1}$, P. Ruffat ${ }^{4}$, F. Cohen-Aubart ${ }^{1,3}, G$. Montalescot ${ }^{2,3}, Z$. Amoura ${ }^{1,3}$. ${ }^{1}$ Service de Médecine interne 2; ${ }^{2}$ Service de Cardiologie, Hopital Pitié Salpétrière; ${ }^{3}$ Sorbonne Université Université Pierre et Marie Curie Paris Vl; ${ }^{4}$ Département d'information médicale, Hopital Pitié Salpétrière, PARIS, France

Background: Coronary artery disease (CAD) is a major cause of morbidity and mortality in systemic lupus erythematosus (SLE) patients. Whether SLE is a cardiovascular risk factor per se remains controversial.

Objectives: This study was conducted to determine the clinical and angiographic characteristics of SLE patients with CAD and to compare them to those of control non-SLE patients with CAD.

Methods: All SLE patients who underwent a coronary angiography procedure in our tertiary centre between 2005 and 2016 were enrolled in the study. Those without significant atherosclerosis (stenosis $>50 \%$ ) were excluded. Each SLE patient was matched by sex and age at catheterization with seven non-SLE controls with significant CAD. Angiographic characteristics were reviewed by two independent cardiologists.

Results: Among the 73 SLE patients who underwent coronary angiography, 28 patients had at least one significant coronary atherosclerotic lesion. The SLE patients were predominantly female $(75 \%$, median age of 55.7 years) with a long standing disease duration (median SLE duration of 20.5 years). Ten patients $(35 \%)$ had renal involvement, and 9 patients $(32 \%)$ had antiphospholipid syndrome. The patients with SLE had fewer cardiovascular risk factors (1.6 vs 2.1 $\mathrm{p}=0.01)$ than the controls, including lower body mass index $\left(23.8 \mathrm{~kg} / \mathrm{m}^{2} \mathrm{vs}\right.$ $24.98 \mathrm{~kg} / \mathrm{m}^{2}, \mathrm{p}=0.03$ ), less frequent family history of coronary artery disease ( $3.5 \%$ vs $18 \%, p=0.049)$ and less diabetes $(7 \%$ vs $22 \%, p=0.07)$ than controls. However, SLE patients were more likely to have chronic kidney failure $(35 \%$ vs $20 \%, \mathrm{p}=0.07$ ) and to need hemodialysis ( $17 \%$ vs $2 \%, \mathrm{p}=0.001)$. The SLE patients more often had multivessel disease $(50 \%)$

Conclusions: While they have fewer cardiovascular risk factors, patients with SLE experience more severe CAD than non-SLE patients, suggesting that SLE, associated conditions or the treatments themselves play key roles in the development of atherosclerosis. ${ }^{2}$

\section{REFERENCES:}

[1] Lee YH, Choi SJ, Ji JD, Song GG. Overall and cause-specific mortality in systemic lupus erythematosus: an updated meta-analysis. Lupus 2016 Jun;25(7):727-734.

[2] Schoenfeld SR, Kasturi S, Costenbader KH. The epidemiology of atherosclerotic cardiovascular disease among patients with SLE: a systematic review. Semin Arthritis Rheum 2013 Aug;43(1):77-95.

Disclosure of Interest: None declared DOI: 10.1136/annrheumdis-2018-eular.5560 